Jonathan D. Emery, MD

Vice Chair, Department of Obstetrics and

Gynecology, Women's Health Institute, Cleveland

Clinic, Cleveland, $\mathrm{OH}$; Assistant Professor,

Cleveland Clinic Lerner College of Medicine of

Case Western Reserve University, Cleveland, $\mathrm{OH}$
Daniel Moussa, BS

Cleveland Clinic Lerner College of Medicine

of Case Western Reserve University,

Cleveland, $\mathrm{OH}$

\title{
Vaccination in pregnancy: A call to all providers for help
}

\section{ABSTRACT}

Vaccination in pregnancy is an important part of maternity care, but maternal immunization rates continue to be below national benchmarks. Influenza and tetanus toxoid, reduced diphtheria toxoid, and acellular pertussis (Tdap) vaccinations have been shown to be safe and provide important protections to pregnant women, the fetus, and neonates. Although obstetrician-gynecologists provide the bulk of pregnancy care, general internists and medical specialists have frequent clinical encounters with maternity patients and should assist in immunization education and administration.

\section{KEY POINTS}

Seasonal influenza and Tdap vaccinations are recommended for women who are pregnant or planning pregnancy.

Both vaccines are safe for most women and offer significant benefits to the mother and baby.

Pregnant women should be given the inactivated form of the influenza vaccine, not the live vaccine.

Tdap vaccination is recommended in the third trimester but can be given at any time in pregnancy.

A recommendation from a healthcare provider is the number-one factor consistently shown to increase maternal vaccination rates.

The COVID-19 vaccine should not be witheld from pregnant patients who meet the criteria for vaccination, but the decision is at the discretion of the patient after an informed discussion.

doi:10.3949/ccjm.88a.20111
$\mathrm{V}$ ACCINATION IN PREGNANCY is a public health priority that is often neglected, even more so today with the current focus on COVID-19 vaccination. Vaccination in pregnancy is important to protect the mother (who is especially vulnerable due to the physiologic changes of pregnancy), fetus (because of developmental and prematurity risks), and neonate (by conferring passive immunity).

This article reviews the 2 vaccines most often given in pregnancy: the inactivated seasonal influenza vaccine and the tetanus toxoid, reduced diphtheria toxoid, and acellular pertussis (Tdap) vaccine, along with evidence of their benefits and safety, guidance on administering them, and management of pregnant women who become sick with or are exposed to influenza or pertussis.

\section{VACCINATION IS RECOMMENDED IN PREGNANCY}

The American College of Obstetricians and Gynecologists (ACOG) advises that not only obstetrician-gynecologists but other healthcare providers recommend and give the influenza vaccine to women who are pregnant or plan to become pregnant during the influenza season. ${ }^{1}$ They also recommend that women be vaccinated against tetanus, diphtheria, and pertussis during every pregnancy. ${ }^{2}$

Despite recommendations from national and international societies, vaccination rates in pregnancy remain lower than targets. ${ }^{3}$ Internists and medical specialists play a critical role in increasing vaccination rates in pregnant women. Because these providers provide ongoing care and surveillance of medical comorbidities such as asthma, diabetes, lupus, and cardiac disease during pregnancy, their action to recommend 
TABLE 1

Summary of vaccinations in pregnancy

\begin{tabular}{lcccc} 
Vaccine & $\begin{array}{c}\text { Indicated } \\
\text { during every } \\
\text { pregnancy }\end{array}$ & $\begin{array}{c}\text { Indicated } \\
\text { for specific } \\
\text { populations } \\
\text { in pregnancy }\end{array}$ & $\begin{array}{c}\text { Contraindicated } \\
\text { in pregnancy }\end{array}$ & $\begin{array}{c}\text { Postpartum } \\
\text { and nursing }\end{array}$ \\
\hline Seasonal influenza & $\sqrt{ }$ & & & $\sqrt{ }$ \\
\hline Tdap & $\sqrt{ }$ & $\sqrt{ }$ & $\sqrt{ }$ \\
\hline Pneumococcal & & $\sqrt{ }$ & $\sqrt{ }$ \\
\hline Hepatitis A & $\sqrt{ }$ & & $\sqrt{ }$ \\
\hline Hepatitis B & & $\sqrt{ }$ & \\
\hline MMR & & $\sqrt{ }$ & \\
\hline Varicella & & & \\
\hline
\end{tabular}

MMR = measles, mumps, rubella; Tdap = tetanus toxoid, reduced diphtheria, and acellular pertussis

and administer immunizations to women who are pregnant or planning a pregnancy is needed to increase vaccination rates.

Table 1 summarizes maternal immunization recommendations.

\section{Vaccination rates in pregnancy remain lower than targets}

\section{INFLUENZA VACCINATION}

Although influenza vaccination rates increased after the $2009 \mathrm{H} 1 \mathrm{~N} 1$ pandemic up to a high of just over 50\% in 2016 in the United States, ${ }^{4}$ rates in pregnancy are declining.
Immunization of pregnant women against seasonal influenza is critically important. During the $2009 \mathrm{H} 1 \mathrm{~N} 1$ influenza pandemic in the United States, 5\% of all influenza deaths were in pregnant women, although pregnant women make up only $1 \%$ of the population. ${ }^{4}$

Influenza in pregnancy places the mother at higher risk of pneumonia, hospitalization, respiratory failure, and intensive care unit admission compared with nonpregnant women. These risks are due to immunologic changes in pregnancy such as altered T-cell immunity and physiologic changes such as the gravid uterus crowding into the chest, causing decreased functional residual capacity of the lungs. Influenza can also lead to preterm labor and birth, potentially subjecting the fetus to the effects of prematurity or causing stillbirth. ${ }^{5}$

\section{Benefits of influenza vaccination for mother and baby}

Vaccination in pregnancy is beneficial for both mother and baby. The effectiveness of seasonal influenza vaccination is similar between pregnant and nonpregnant women. ${ }^{6}$ Although the efficacy of the seasonal vaccine varies from year to year, pregnant women who have been vaccinated have fewer hospital admissions than those not vaccinated. ${ }^{1,4}$

Vaccinating the mother can also have substantial benefits for the newborn after birth, owing to passive immunity conferred by transplacental passage of maternal antibodies. A randomized study found umbilical cord blood antibody titers to be more than 1.5 times higher than those in maternal blood after vaccination. ${ }^{7}$ Clinical benefits include fewer hospitalizations and lower rates of laboratory-confirmed influenza illness in infants born to vaccinated mothers. In 2 studies, 93 of 2,873 (3.24\%) infants of vaccinated women developed laboratoryconfirmed influenza illness compared with 142 of 2,869 (4.95\%) infants of unvaccinated women (risk ratio 0.66, 95\% confidence interval 0.50-0.85). ${ }^{8}$ From these data we calculate the absolute risk reduction as $1.71 \%$ and the number needed to treat 58 . 


\section{No evidence of safety concerns}

Numerous studies have found influenza vaccination to be safe and effective in pregnancy, with few adverse effects on the mother. ${ }^{1,4,5}$ Common vaccine-related side effects include injection site reaction, fever, headache, and myalgia.

Possible adverse effects of the seasonal flu vaccine on the fetus and neonate have also been studied. Systematic reviews of maternal inactivated influenza virus vaccination have found no correlation with increased fetal risk. ${ }^{9,10}$ A prospective cohort study of more than 10,000 pregnant women found no association between influenza vaccination in pregnancy and adverse birth outcomes. ${ }^{11} \mathrm{Al}$ though concerns have been raised about the safety of the mercury-containing preservative thimerosal and its possible links to autism, no scientific evidence of a link between receipt of thimerosal-containing vaccines in the mother and adverse health or developmental effects in the baby has ever been found. ${ }^{1}$

\section{Administration recommendations}

Only the inactivated influenza vaccine should be used for pregnant women. The live, attenuated influenza vaccine is indicated for nonpregnant patients ages 2 to 49 , and it may be used for postpartum women, including those who are breastfeeding.

Contraindications. A prior life-threatening reaction to the influenza vaccine or to any of its components is an absolute contraindication to receiving the vaccine. Even for women with a history of egg allergy or Guillain-Barré syndrome, vaccination should be considered if the potential benefits outweigh the risks. ${ }^{12}$

Timing. Pregnant women can receive the inactivated influenza vaccine at any time during influenza season (typically October through May), although the best time is early in the season, ideally before the end of October. An unvaccinated pregnant woman presenting to her primary care provider or medical specialist should be encouraged to receive the vaccine regardless of her stage of pregnancy.

Women of reproductive age who are planning a pregnancy during flu season or are not actively using contraception should be given the vaccine.
Documentation of vaccination should be provided to the patient to ensure her immunization records are kept current.

Family members of pregnant women should also be vaccinated. Although this strategy is recommended to reduce illness in newborns, data are mixed about its efficacy. ${ }^{5}$

\section{Treating influenza and possible exposure}

Whether or not they have been vaccinated, women who develop flulike illness at any time during pregnancy should be prescribed antiviral medications, ie, one of the following regimens, depending on local influenza virus resistance ${ }^{13}$ :

- Oseltamivir $75 \mathrm{mg}$ twice daily for 5 days (recommended by the US Centers for Disease Control and Prevention)

- Zanamivir $10 \mathrm{mg}$ (2 5-mg inhalations) twice daily for 5 days

- Peramivir 600 mg (single intravenous infusion).

Treatment should be initiated as soon as possible after onset of illness.

Postexposure antiviral prophylaxis should be considered for pregnant and postpartum women, with either of the following regimens:

- Oseltamivir $75 \mathrm{mg}$ once daily for 7 to 10 days

- Zanamivir $10 \mathrm{mg}$ (2 5-mg inhalations) once daily for 10 days).

Therapy should be given presumptively without laboratory confirmation if suspicion is high for pregnant women and women who are up to 2 weeks postpartum, including women who have experienced a fetal loss.

\section{TDAP VACCINATION}

Tdap vaccination is recommended during pregnancy by the Advisory Council on Immunization Practices (ACIP) and ACOG, primarily to help protect neonates against the highly contagious Bordetella pertussis bacteria. ${ }^{14}$ Despite that, the rate of Tdap immunization in pregnancy hovers just over $50 \%$.

Infants younger than 1 year are at the highest risk of severe symptoms and sequelae from pertussis; it is a major cause of vaccinepreventable death in this age group. ${ }^{15}$

Vaccination is highly effective in protecting infants against pertussis infection, preventing over $90 \%$ of hospitalizations and $95 \%$

\section{Only the inactivated influenza vaccine should be used for pregnant women}


of deaths due to pertussis in the first 2 to 3 months of life. ${ }^{16}$ Vaccination during pregnancy is especially important, as it bolsters maternal pertussis-specific IgG antibodies, which are passively acquired by the fetus during pregnancy. ${ }^{17}$ Without maternal vaccination, there is a gap in protection after delivery, as infants are unable to receive their first pertussis vaccination until 6 weeks of age, when they start their 5 -dose course at set intervals between 2 months and 6 years of age. ${ }^{14}$

\section{Timing of Tdap immunization}

Tdap should be given to pregnant women at the beginning of the third trimester of each pregnancy, ideally between 27 and 36 weeks gestation, regardless of timing of prior Tdap vaccination. It can be given earlier in pregnancy for maternal benefit (such as tetanus exposure), and in such cases, does not need to be repeated later in pregnancy or postpartum. For women who did not receive Tdap prenatally, it can be given postpartum. It should not be given preconception unless the patient is due for her scheduled vaccination.

Pertussis vaccination is contraindicated for patients who have had a severe allergic reac-
Pregnant women who develop flulike illness should be prescribed antiviral medications tion after a previous dose or encephalopathy without identifiable cause within 7 days of receiving a pertussis vaccine.

\section{Vaccinating family members}

Since 2005, the ACIP has recommended a strategy of "cocooning" (ie, vaccinating people who come into close contact with a newborn) to provide the best protection against pertussis until the recommended childhood vaccination schedule can begin. ${ }^{14}$ However, programs using this strategy have had inconsistent results due to challenges in vaccinating other family members. Also, evidence suggests that immunization with Tdap may not fully prevent bacterial transmission. Nevertheless, the ACIP continues to recommend this practice, and primary care physicians, who may care for multiple members of a family, may be able to promote this policy.

\section{Addressing safety concerns}

The maternal and fetal safety of Tdap vaccination has been well documented, ${ }^{18}$ and it does not increase pregnancy risks such as preeclampsia, fetal growth restriction, stillbirth, or preterm birth. ${ }^{19}$ No fetal or neonatal developmental risks have been identified. Similar to the seasonal influenza vaccine, common reactions to maternal Tdap administration include pain at the injection site, headache, and fatigue.

\section{Treating pertussis exposure}

Although pertussis is primarily managed through vaccination in the United States, infection does occur. Pertussis has 3 stages: a catarrhal stage with upper respiratory infection symptoms, a paroxysmal stage with coughing spasms, and a convalescent stage with slow resolution of coughing frequency and severity. Patients are most infectious during the catarrhal and paroxysmal stages. ${ }^{20}$

If a pregnant patient or a household contact has been exposed to pertussis, treatment should begin within 21 days of exposure to reduce symptomatic infection and spread. Treatment for pregnant adults is with one of the following regimens ${ }^{21}$ :

- Azithromycin $500 \mathrm{mg}$ in a single dose on day 1 , then $250 \mathrm{mg}$ per day on days 2 through 5

- Erythromycin 2 g per day in 4 divided doses for 14 days.

Treatment of the mother is especially important during the postpartum period to reduce the risk of spread to the newborn. Either regimen is safe during lactation.

\section{ALL PHYSICIANS SHOULD PROMOTE VACCINATION}

Primary care providers and medical specialists should routinely assess the vaccination status of their patients, especially pregnant women, and recommend and administer appropriate vaccines. Although obstetrician-gynecologists bear primary responsibility for providing and administering vaccinations to pregnant patients, not all carry influenza or Tdap vaccines in their offices due to financial or logistical reasons, such as storage or tracking of vaccines. ${ }^{22}$ A national US survey of obstetriciangynecologists found that for those whose practice did not stock an indicated vaccine, 56\% "always or often" referred patients to their primary care provider to receive the vaccine, referring less often to public health departments $(32 \%)$ or pharmacies $(25 \%) .{ }^{22}$ 
Many pregnant women refuse the Tdap and influenza vaccines ${ }^{23}$ for a variety of reasons, ${ }^{24}$ including common misperceptions ("It will make me sick"), concerns for the safety of the fetus or neonate, and personal health beliefs ("I never get the flu shot"). A review by Mossad ${ }^{25}$ provides guidance on counseling patients who doubt the value of immunization.

Studies of various vaccine promotion techniques, such as text messaging or video tutorials, have found that they result in only modest, if any, increase in maternal vaccination acceptance. ${ }^{26,27}$ Multiple studies have shown that the recommendation of a healthcare provider is the single most influential factor of pregnant women accepting vaccinations in pregnancy. ${ }^{4,23,28}$

\section{COVID-19 VACCINATION IN PREGNANCY}

In December 2020, the US Food and Drug Administration issued an Emergency Use Authorization for 2 of the vaccines against SARS-CoV-2 (COVID-19).

Soon after, $\mathrm{ACOG}^{29}$ issued a statement about COVID-19 vaccination in pregnancy, stating, "ACOG recommends that COVID-19 vaccines should not be withheld from pregnant individuals who meet criteria for vaccination based on ACIP-recommended priority groups.” The US Centers for Disease Control and Prevention (CDC) issued a similar statement. ${ }^{30}$ It is recommended, but not required, that pregnant and lactating patients have a discussion with their provider about their own specific circumstances and risks and whether to accept the vaccine.

While no pregnant patients were included in critical vaccine safety trials, it is known that a pregnant woman who contracts COVID-19 is at higher risk of complications compared with her nonpregnant counterparts, ${ }^{31}$ and the CDC has responded by including pregnancy as a risk factor for a high-risk health group. As such, pregnant patients, especially those in high-risk occupations, should be offered the vaccine after appropriate counseling as set forth by guidance from ACOG and the CDC.

However, the decision to be vaccinated is solely at the patient's discretion, and a conversation with her healthcare team about her decision to be vaccinated is recommended but not required. Finally, clinicians are encouraged to keep abreast of available evidence about the COVID-19 vaccines in terms of risks and benefits, as well about potential complications, so that they can provide the most accurate information and counseling to their patients.

\section{DISCLOSURES}

The authors report no relevant financial relationships which, in the context of their contributions, could be perceived as a potential conflict of interest.

\section{REFERENCES}

1. ACOG Committee Opinion No. 732: influenza vaccination during pregnancy. Obstet Gynecol 2018; 131(4):e109-e114. doi:10.1097/AOG.0000000000002588

2. ACOG Committee Opinion No. 741: maternal immunization. Obstet Gynecol 2018; 131(6):e214-e217. doi:10.1097/AOG.0000000000002662

3. Sakala IG, Honda-Okubo Y, Fung J, Petrovsky N. Influenza immunization during pregnancy: benefits for mother and infant. Hum Vaccin Immunother 2016; 12(12):3065-3071. doi:10.1080/21645515.2016.1215392

4. Rasmussen SA, Jamieson DJ. Influenza and pregnancy: no time for complacency. Obstet Gynecol 2019; 133(1):23-26. doi:10.1097/AOG.0000000000003040

5. Marshall H, McMillan M, Andrews RM, Macartney K, Edwards K. Vaccines in pregnancy: the dual benefit for pregnant women and infants. Hum Vaccin Immunother 2016; 12(4):848-856. doi:10.1080/21645515.2015.1127485

6. Thompson MG, Li DK, Shifflett P, et al. Effectiveness of seasonal trivalent influenza vaccine for preventing influenza virus illness among pregnant women: a population-based case-control study during the 2010-2011 and 2011-2012 influenza seasons. Clin Infect Dis 2014; 58(4):449-457. doi:10.1093/cid/cit750

7. Vesikari T, Virta M, Heinonen S, et al. Immunogenicity and safety of a quadrivalent inactivated influenza vaccine in pregnant women: a randomized, observer-blind trial. Hum Vaccin Immunother 2020; 16(3):623-629. doi:10.1080/21645515.2019.1667202

8. Jarvis JR, Dorey RB, Warricker FDM, Alwan NA, Jones CE. The effectiveness of influenza vaccination in pregnancy in relation to child health outcomes: systematic review and meta-analysis. Vaccine 2020; 38(7):1601-1613. doi:10.1016/j.vaccine.2019.12.056

9. Bednarczyk RA, Adjaye-Gbewonyo D, Omer SB. Safety of influenza immunization during pregnancy for the fetus and the neonate. Am J Obstet Gynecol 2012; 207(3 suppl):S38-S46. doi:10.1016/j.ajog.2012.07.002

10. Giles ML, Krishnaswamy S, Macartney K, Cheng A. The safety of inactivated influenza vaccines in pregnancy for birth outcomes: a systematic review. Hum Vaccin Immunother 2019; 15(3):687-699. doi:10.1080/21645515.2018.1540807

11. Ohfuji S, Deguchi M, Tachibana D, et al. Safety of influenza vaccination on adverse birth outcomes among pregnant women: a prospective cohort study in Japan. Int J Infect Dis 2020; 93:68-76. doi:10.1016/j.ijid.2020.01.033

12. Psarris A, Sindos M, Daskalakis G, et al. Immunizations during pregnancy: how, when and why. Eur J Obstet Gynecol Reprod Biol 2019; 240:29-35. doi:10.1016/j.ejogrb.2019.06.019

13. ACOG Committee Opinion No. 753 Summary: assessment and treatment of pregnant women with suspected or confirmed influenza. 
Obstet Gynecol 2018; 132(4):1077-1079. doi:10.1097/AOG.0000000000002873

14. Liang JL, Tiwari T, Moro $\mathbf{P}$, et al. Prevention of pertussis, tetanus, and diphtheria with vaccines in the United States: recommendations of the Advisory Committee on Immunization Practices (ACIP). MMWR Recomm Rep 2018; 67(2):1-44. doi:10.15585/mmwr.rr6702a1

15. Vittucci AC, Spuri Vennarucci V, Grandin A, et al. Pertussis in infants: an underestimated disease. BMC Infect Dis 2016; 16(1):414. doi:10.1186/s12879-016-1710-0

16. Smallenburg LC, van Welie NA, Elvers LH, van Huisseling JC, Teunis PF, Versteegh FG. Decline of IgG pertussis toxin measured in umbilical cord blood, and neonatal and early infant serum. Eur J Clin Microbiol Infect Dis 2014; 33(9):1541-1545. doi:10.1007/s10096-014-2110-2

17. Forsyth K, Plotkin S, Tan T, Wirsing von König CH. Strategies to decrease pertussis transmission to infants. Pediatrics 2015; 135(6):e1475-e1482. doi:10.1542/peds.2014-3925

18. McMillan M, Clarke M, Parrella A, Fell DB, Amirthalingam G, Marshall HS. Safety of tetanus, diphtheria, and pertussis vaccination during pregnancy: a systematic review. Obstet Gynecol 2017; 129(3):560-573. doi:10.1097/AOG.0000000000001888

19. Furuta M, Sin J, Ng ESW, Wang K. Efficacy and safety of pertussis vaccination for pregnant women-a systematic review of randomised controlled trials and observational studies. BMC Pregnancy Childbirth 2017; 17(1):390. doi:10.1186/s12884-017-1559-2

20. Tiwari T, Murphy TV, Moran J; National Immunization Program, CDC. Recommended antimicrobial agents for the treatment and postexposure prophylaxis of pertussis: 2005 CDC Guidelines. MMWR Recomm Rep 2005; 54(RR-14):1-16. pmid:16340941

21. Healy CM. Pertussis vaccination in pregnancy. Hum Vaccin Immunother 2016; 12(8):1972-1981. doi:10.1080/21645515.2016.1171948

22. O'Leary ST, Riley LE, Lindley MC, et al. Immunization practices of US obstetrician/gynecologists for pregnant patients. Am J Prev Med 2018; 54(2):205-213. doi:10.1016/j.amepre.2017.10.016

23. Ahluwalia IB, Jamieson DJ, Rasmussen SA, D'Angelo D, Goodman D, Kim H. Correlates of seasonal influenza vaccine coverage among pregnant women in Georgia and Rhode Island. Obstet Gynecol 2010; 116(4):949-955. doi:10.1097/AOG.0b013e3181f1039f

24. O'Leary ST, Riley LE, Lindley MC, et al. Obstetrician-gynecologists' strategies to address vaccine refusal among pregnant women. Obstet Gynecol 2019; 133(1):40-47. doi:10.1097/AOG.0000000000003005

25. Mossad SB. How to respond to flu vaccine doubters. Cleve Clin J Med 2019; 86(12):782-788. doi:10.3949/ccjm.86a.19139

26. Yudin $\mathbf{M H}$, Mistry N, De Souza LR, et al. Text messages for influenza vaccination among pregnant women: a randomized controlled trial. Vaccine 2017; 35(5):842-848. doi:10.1016/j.vaccine.2016.12.002

27. Goodman K, Mossad SB, Taksler GB, Emery J, Schramm S, Rothberg MB. Impact of video education on influenza vaccination in pregnancy. J Reprod Med 2015; 60(11-12):471-479. pmid:26775454

28. Shavell VI, Moniz MH, Gonik B, Beigi RH. Influenza immunization in pregnancy: overcoming patient and health care provider barriers. Am J Obstet Gynecol 2012; 207(3 suppl):S67-S74. doi:10.1016/j.ajog.2012.06.077

29. American College of Obstetricians and Gynecolgists. Vaccinating pregnant and lactating patients against COVID-19. Practice advisory. December 2020. Accessed February 12, 2021. https://www. acog.org/en/Clinical/Clinical\%20Guidance/Practice \%20Advisory/ Articles/2020/12/Vaccinating \%20Pregnant $\% 20$ and $\% 20$ Lactating $\% 20$ Patients\%20Against\%20COVID\%2019

30. Centers for Disease Control and Prevention. COVID-19 vaccination considerations for people who are pregnant. Updated January 2 7, 2021. Accessed February 12, 2021. https://www.cdc.gov/ coronavirus/2019-ncov/vaccines/recommendations/pregnancy.html

31. Ellington S, Strid P, Tong VT, et al. Characteristics of women of reproductive age with laboratory-confirmed SARS-CoV-2 infection by pregnancy status - United States, January 22-June 7, 2020. MMWR Morb Mortal Wkly Rep 2020; 69(25):769-775 doi:http://dx.doi.org/10.15585/mmwr.mm6925a1

Address: Jonathan D. Emery, MD, Department of Obstetrics and Gynecology, WH20, Cleveland Clinic, 2570 SOM Center Road, Willoughby Hills, $\mathrm{OH}$ 44094; emeryj@ccf.org 